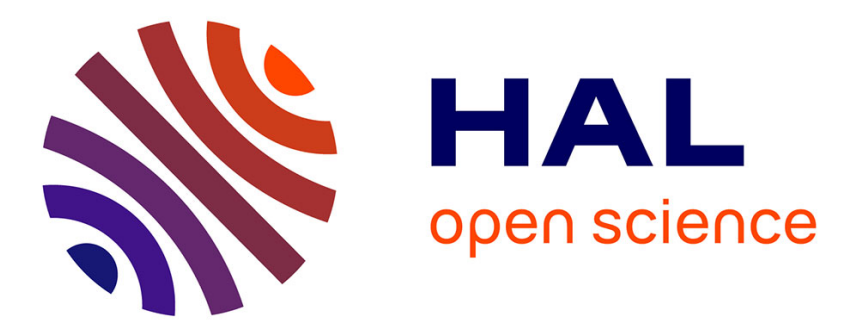

\title{
Image Compression Through a Projection onto a Polyhedral Set
}

François Malgouyres

\section{To cite this version:}

François Malgouyres. Image Compression Through a Projection onto a Polyhedral Set. Journal of Mathematical Imaging and Vision, 2007, 11, pp.670 - 200. 10.1007/s10851-006-9000-x . hal01486817

\section{HAL Id: hal-01486817 https://hal.science/hal-01486817}

Submitted on 10 Mar 2017

HAL is a multi-disciplinary open access archive for the deposit and dissemination of scientific research documents, whether they are published or not. The documents may come from teaching and research institutions in France or abroad, or from public or private research centers.
L'archive ouverte pluridisciplinaire HAL, est destinée au dépôt et à la diffusion de documents scientifiques de niveau recherche, publiés ou non, émanant des établissements d'enseignement et de recherche français ou étrangers, des laboratoires publics ou privés. 


\title{
Image compression through a projection onto a polyhedral set
}

\author{
F. Malgouyres*
}

\begin{abstract}
In image denoising, many researchers have tried for several years to combine wavelet-like approaches and optimization methods (typically based on the total variation minimization). However, despite the well-known links between image denoising and image compression when solved with wavelet-like approaches, these hybrid image denoising methods have not found counterparts in image compression. This is the gap that this paper aims at filling. To do so, we provide a generalization of the standard image compression model. However, important numerical limitations still need to be addressed in order to make such models practical.
\end{abstract}

\section{Introduction}

\subsection{Recollections on image compression}

At the core of the usual image compression standards (for instance JPEG and JPEG 2000) is a rounding procedure. Given a basis $\mathcal{B}=\left(\Psi_{i}\right)_{i \in I}$ of $\mathbb{R}^{N}$, this rounding is applied to the coordinates of the image $u \in \mathbb{R}^{N}$, with respect to this basis. More precisely, given a quantization table ${ }^{1}\left(\tau_{i, k}\right)_{i \in I, k \in \mathbb{Z}}$, such that, for all $i \in I$ and $k \in \mathbb{Z}, \tau_{i, k}<\tau_{i, k+1}$, and $u=\sum_{i \in I} U_{i} \Psi_{i}$, the coder only describes $\left(k_{i}\right)_{i \in I}$, where, for all $i \in I$, $k_{i}$ is such that

$$
\tau_{i, k_{i}} \leq U_{i}<\tau_{i, k_{i}+1}
$$

Moreover, if we assume ${ }^{2} \tau_{i, 0}<0<\tau_{i, 1}$, for all $i \in I$, one just needs to code the non-zero indexes as well as their location. Indeed, for an image $u=\sum_{i \in I} U_{i} \Psi_{i} \in \mathbb{R}^{N}$, denoting

$$
J=\left\{i \in I, U_{i}<\tau_{i, 0} \text { or } \tau_{i, 1} \leq U_{i}\right\}
$$

coders usually encode $\left(J,\left(k_{j}\right)_{j \in J}\right)$. It is indeed often cheaper to encode $J$ and the corresponding coefficients than the sequence with the zeros.

Roughly speaking, the codelength is proportional to \#J, where \# denotes the cardinal of a finite set. However, the ratio between the codelength and \#J plays an important role and depends on the basis used to compress the image. For instance, a wavelet basis allows tree coding. The ratio between the codelength and $\# J$ is then smaller than with many other bases.

Leaving this issue aside, the goal in image compression has been to find a basis such that \# $J$ is as small as possible. Many bases have been proposed to represent images sparsely (see, for instance, [11, 24]) or a particular image content (for example, for textures, see [7, 19]). The JPEG and JPEG 2000 standards use a local cosine and a wavelet basis respectively (e.g., see [17]).

Bases are usually rated according to the decay of the sequence $\left(\left|U_{i}\right|\right)_{i \in I}$, after it has been sorted in decaying order. For arguments in favor of wavelet bases, see for example [20], where Besov semi-norms are characterized by wavelet coefficients and [5], where the space of bounded variation is studied under the scope of wavelet coefficients decay.

Besides basis design, which has been a very active field in the past ten years, the research in image compression is currently following other paths. Examples can be found in $[4,18,8,21,23,10,3]$.

\footnotetext{
* LAGA/L2TI, Université Paris 13, 99 avenue Jean-Batiste Clément, 93430 Villetaneuse, France.

${ }^{1}$ If $\tau_{i, k}=k \tau_{i}$, for a sequence $\left(\tau_{i}\right)_{i \in I}$, the rounding process described is a quantization. We will therefore abusively use the term "quantization table" for the characteristics of the rounding process.

${ }^{2}$ This is not a serious restriction since $k_{i}=0$ plays a central role in image compression and $k_{i}=0$ usually corresponds to coordinates whose modulus is small.
} 
Notice that all these methods define a partition of $\mathbb{R}^{N}$ : the partition is given by the equivalence classes of the relation defined by $u \sim v$ if and only if $u$ and $v$ provide the same code. It is of course crucial that the partition "agrees" with the kind of information contained in images.

For image compression standards, the elements of the partition are polyhedra. They are characterized by a sequence $\left(k_{i}\right)_{i \in I}$ and are defined by

$$
\mathcal{C}_{\left(k_{i}\right)_{i \in I}}=\left\{u=\sum_{i \in I} U_{i} \Psi_{i}, \forall i \in I, \tau_{i, k_{i}} \leq U_{i}<\tau_{i, k_{i}+1}\right\} .
$$

In their simplest form, the results of this paper aim at giving other possible characterization of $\mathcal{C}_{\left(k_{i}\right)_{i \in I}}$. This characterization is determined by solving a constrained optimization problem : the latter consists of minimizing a function $f(v)$ under the constraint $v \in \overline{\mathcal{C}}_{\left(k_{i}\right)_{i \in I}}$ (the bar denotes the closure). In this sense, it is a projection onto $\overline{\mathcal{C}}_{\left(k_{i}\right)_{i \in I}}$. In Section 2, we will show that $\mathcal{C}_{\left(k_{i}\right)_{i \in I}}$ is characterized by the active constraint $^{3}$ to the proposed optimization problem.

The potential interests of this characterization are the following :

- It generalizes the usual coding procedure : as shown in Section 3.2, the coding of $\left(J,\left(k_{j}\right)_{j \in J}\right)$ corresponds to a particular choice for $f$. Other choices might provide smaller characterizations.

- There should be many other possibilities for the choice of partition defining the compression algorithm. We have not investigated this aspect, but it should also lead to a significant generalization of the usual coders.

- It separates the building of the partition (which should be built with regard to image resemblance only) and the coding of the elements of the partition. Ideally, small codes should be reserved for elements of the partition containing images. (Those which do not contain images should have large codes.) This is not the case for many codes with the standard coders! For instance, an element of $\mathbb{R}^{N}$ built with only a few non-zero random coordinates has a very small code and is very unlikely to represent an image.

However, the method will remain of limited practical interest unless the associated optimization problems (one for compression and one for reconstruction) can be solved exactly and rapidly. In practice, an analytical solution to the optimization problems probably needs to be available. This is of course a very strong restriction on the choice of the partition and the function $f$. As far as we know, this leaves us with partitions and functions $f$ built with coordinates in a basis. Future development in numerical analysis might allow more complex models though (see, for instance, [9] where unexpected numerical analysis results are exposed).

Also, it should be noted that the kinds of optimization problems that need to be solved to compress and decompress images have been considered with respect to the restoration of compressed images (see [6, 1]). However, we would like to stress that it is not the purpose of this paper to restore compressed images : the proposed decompression procedure aims at providing the whole element of the partition containing the image. Any restoration algorithm can then be used to improve the image.

\subsection{Links with image denoising}

In image denoising, a datum $u \in \mathbb{R}^{N}$ is assumed to be given in the form

$$
u=v+b,
$$

where $b$ is a Gaussian noise and $v$ is the ideal image to be recovered. (The Gaussian noise assumption is, of course, a rough approximation to practical denoising problems.) Wavelet-like approaches to image denoising use the fact that the noise, whatever orthonormal basis it is expressed in, remains a Gaussian noise : it is therefore spread over many small coefficients. The goal is therefore to find a basis which

\footnotetext{
${ }^{3}$ The constraint $i$ is called active if the solution $v^{\prime}=\sum_{i \in I} v_{i}^{\prime} \Psi_{i}$ to the optimization problem satisfies either $v_{i}^{\prime}=\tau_{i, k_{i}}$ or $v_{i}^{\prime}=\tau_{i, k_{i}+1}$.
} 
concentrates the content of the ideal image $v$ over a few large coefficients. Then, when cancelling small coefficients of $u$, one cancels most of the noise and keeps most of the information.

The goal is again to find a basis providing a sparse representation of images : the decay of the sorted coordinates modulus of natural images in the basis is the key argument which guarantees the success of the method. That is (in short) why, in the wavelet community, image compression and image denoising are considered similar problems.

In this respect, the model we will describe for image compression is closely related to a model which has been studied successively in $[6,14,2,13,12]$. (The respective contributions of these paper is detailed in [12].) In its most general form, it consists of

$$
\left\{\begin{array}{l}
\text { minimizing } f(v) \\
\text { under the constraints, }\langle H(v)-u, \Psi\rangle \leq \tau, \text { for all } \Psi \in \mathcal{D},
\end{array}\right.
$$

for a finite dictionary $\mathcal{D}$ of elements of $\mathbb{R}^{N}$, a linear operator $H$, a datum $u \in \mathbb{R}^{N}$ and a function $f$, convex over $\mathbb{R}^{N}$ (typically, the total variation).

The link between the restoration and compression models is that they both use the same representation of $\mathbb{R}^{N}$; we show in [15] that they implicitly define a parameterization of $\mathbb{R}^{N}$. This parameterization is defined through an optimization model, characterized by a pair $f / \mathcal{D}$, and replaces the usual characterization of elements of $\mathbb{R}^{N}$ as linear expansions.

Although the current paper deals with image compression, it gives a strong insight on denoising models of the (1). It indeed suggests that the couple $f / \mathcal{D}$ defining (1) should be chosen so that the number of active constraints is small when the initial datum is an image. We would like to emphasize that at present, no theoretical or practical arguments are available to chose the pair $f / \mathcal{D}$.

\subsection{Organization of the paper}

In Section 2, we state the optimization results which justify the proposed method; we describe the compression/decompression process in Section 3 and sketch the computations that need to be achieved in order to compress and decompress an image. We also show that the proposed process is a generalization of the standard compression/decompression schemes in Section 3. Finally, some concluding remarks are given in Section 4 .

\section{Mathematical facts}

\subsection{Two equivalent optimization problems}

Theorem 1 Let $N>0$ be an integer, $f$ be a strictly convex function over $\mathbb{R}^{N}$ and $\mathcal{D}=\left(\Psi_{i}\right)_{i \in I}$ be a finite set of non-zero elements of $\mathbb{R}^{N}$. Let $\boldsymbol{\tau}=\left(\left(\tau_{i,-}, \tau_{i,+}\right)\right)_{i \in I} \in \mathbb{R}^{2 I}$ and

$$
\mathcal{C}=\left\{v \in \mathbb{R}^{N}, \forall i \in I, \tau_{i,-} \leq\left\langle v, \Psi_{i}\right\rangle \leq \tau_{i,+}\right\}
$$

Also assume that $\mathcal{D}$ and $\boldsymbol{\tau}$ are such that $\mathcal{C}$ has non-empty interior and is bounded. The problem

$$
(P):\left\{\begin{array}{l}
\text { minimize } f(v), \\
\text { over, } v \in \mathcal{C} .
\end{array}\right.
$$

has a unique solution, $Q$. Writing $J=\left\{i \in I,\left\langle Q, \Psi_{i}\right\rangle=\tau_{i,-}\right.$ or $\left.\left\langle Q, \Psi_{i}\right\rangle=\tau_{i,+}\right\}$, and $\tau_{j}=\left\langle Q, \Psi_{j}\right\rangle$, for $j \in J, Q$ is also the unique solution to

$$
\left(P^{\prime}\right):\left\{\begin{array}{l}
\text { minimize } f(v) \\
\text { over, } v \in \mathcal{C}^{\prime}
\end{array}\right.
$$

with

$$
\mathcal{C}^{\prime}=\left\{v \in \mathbb{R}^{N}, \forall j \in J,\left\langle v, \Psi_{j}\right\rangle=\tau_{j}\right\} .
$$


Proof.

First, $(P)$ obviously has a unique solution $Q \in \mathbb{R}^{N}$ and a Kuhn-Tucker vector $\boldsymbol{\lambda}=\left(\left(\lambda_{i,-}, \lambda_{i,+}\right)\right)_{i \in I}$ (see [22], Th. 27.3, pp.267 and Cor 28.2.1, pp 278). Therefore (see [22], Th. 28.3, pp 281), $(Q, \boldsymbol{\lambda})$ satisfies

$$
\left\{\begin{array}{l}
\lambda_{i,-} \geq 0,\left\langle Q, \Psi_{i}\right\rangle \geq \tau_{i,-} \text { and } \lambda_{i,-}\left(\left\langle Q, \Psi_{i}\right\rangle-\tau_{i,-}\right)=0, \text { for all } i \in I, \\
\lambda_{i,+} \geq 0,\left\langle Q, \Psi_{i}\right\rangle \leq \tau_{i,+} \text { and } \lambda_{i,+}\left(\left\langle Q, \Psi_{i}\right\rangle-\tau_{i,+}\right)=0, \text { for all } i \in I \\
\text { and } 0 \in \partial f(Q)+\sum_{i \in I}\left(\lambda_{i,+}-\lambda_{i,-}\right) \Psi_{i} .
\end{array}\right.
$$

This obviously implies that, for all $i \notin J, \lambda_{i,-}=\lambda_{i,+}=0$ and therefore

$$
\left\{\begin{array}{l}
\left\langle Q, \Psi_{j}\right\rangle=\tau_{j}, \text { for all } j \in J \\
\text { and } 0 \in \partial f(Q)+\sum_{j \in J}\left(\lambda_{j,+}-\lambda_{j,-}\right) \Psi_{j}
\end{array}\right.
$$

This implies that $Q$ is a solution to $\left(P^{\prime}\right)$ (again, using Th. 28.3 in [22], pp 281.)

This result is illustrated by Figure 1, it means that $Q$ can be deduced from either $(P)$ or $\left(P^{\prime}\right)$. Notice that $\left(P^{\prime}\right)$ is described by $\left(J,\left(\tau_{j}\right)_{j \in J}\right)$, if we assume $f$ and $\left(\Psi_{i}\right)_{i \in I}$ are known.

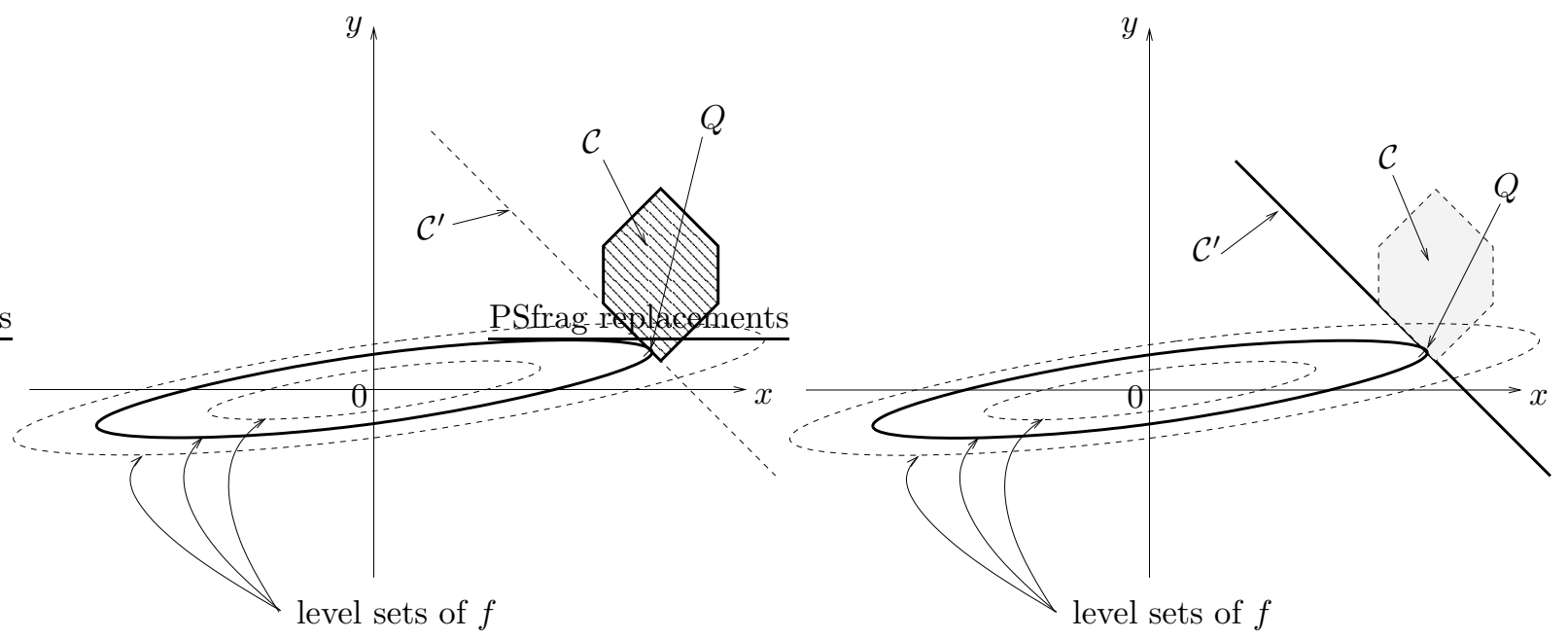

Figure 1: In 2D : Left : $Q$ achieves the minimum of $f$ among points of the hexagon $\mathcal{C}$. Right : $Q$ achieves the minimum of $f$ among points of the line $\mathcal{C}^{\prime}$.

\subsection{Coding a set through a solution to an optimization problem}

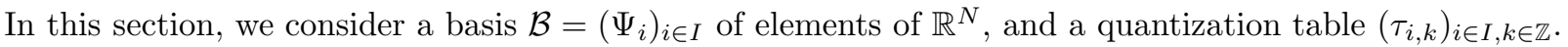
We also consider a partition of $\mathbb{R}^{N}$ whose elements $\mathcal{C}$ are defined by a sequence $\left(k_{i}\right)_{i \in I}$ and are such that the closure of $\mathcal{C}$ is given by

$$
\overline{\mathcal{C}}=\left\{v=\sum_{i \in I} V_{i} \Psi_{i}, \text { for all } i \in I, \tau_{i, k_{i}} \leq V_{i} \leq \tau_{i, k_{i}+1}\right\},
$$

and its interior by

$$
\stackrel{\circ}{\mathcal{C}}=\left\{v=\sum_{i \in I} V_{i} \Psi_{i}, \text { for all } i \in I, \tau_{i, k_{i}}<V_{i}<\tau_{i, k_{i}+1}\right\} .
$$

In the remainder of the paper, we will use the word partition to describe such a partition.

Let us also isolate a necessary property of the partition in the following definition.

Definition 1 Let $f$ be a continuously differentiable, strictly convex function over $\mathbb{R}^{N}$. A partition is called $f$-proper if, for any element $\mathcal{C}$ of the partition characterized by $\left(k_{i}\right)_{i \in I}$, the solution $Q=\sum_{i \in I} q_{i} \Psi_{i}$ to $(P)$ satisfies either

$$
\tau_{i, k_{i}}<q_{i}<\tau_{i, k_{i}+1}
$$




$$
\lambda_{i} \neq 0
$$

(but not both), where

$$
\nabla f(Q)+\sum_{i \in I} \lambda_{i} \Psi_{i}=0
$$

The property f-proper means that the active constraints of a solution to $(P)$ are actually active; this rules out the possibility that $q_{i}=\tau_{i, k_{i}}$ or $q_{i}=\tau_{i, k_{i}+1}$ occurs by chance. As is shown in the proof of Theorem 4 in [15], the prohibited situation only occurs when 0 belongs to a set whose Lebesgue measure is zero; it is therefore very unlikely. If this happens however, a small perturbation of the $\tau$ values should allow the condition to be met without significantly modifying the partition.

In image compression, the refinement of the partition is an important property. In this respect, we will say that $f$ is homogeneous if there exists $\alpha>0$ such that, for any $\beta>0$ and any $u \in \mathbb{R}^{N}$,

$$
f(\beta u)=\beta^{\alpha} f(u)
$$

Proposition 1 Let $f$ be an homogeneous, continuously differentiable, strictly convex function over $\mathbb{R}^{N}$ and let $\mathcal{P}$ be an $f$-proper partition. Consider $\beta>0$ and the partition $\beta \mathcal{P}$ whose elements are of the form $\beta \mathcal{C}$, for $\mathcal{C}$ in $\mathcal{P}$. The partition $\beta \mathcal{P}$ is $f$-proper.

Proof. First observe that, for any $u \in \mathbb{R}^{N}$,

$$
\nabla f(\beta u)=\beta^{\alpha-1} \nabla f(u)
$$

Therefore (see (2)), if we denote by $Q$ the solution to $(P), \beta Q$ is a solution to

$$
\left\{\begin{array}{l}
\text { minimize } f(v), \\
\text { over, } v \in \overline{\beta \mathcal{C}} .
\end{array}\right.
$$

and its Kuhn-Tucker vector is $\beta^{\alpha-1} \boldsymbol{\lambda}$, where $\boldsymbol{\lambda}$ is the Kuhn-Tucker vector to $(P)$. This guaranties that the proposition holds.

The next theorem states that there is a one-to-one correspondence between an element of an $f$-proper partition and the solution to the optimization problem defined by $(P)$. Using the above notation, it is therefore possible to code an element $\mathcal{C}$ of the partition by coding $Q$.

Theorem 2 Let $f$ be a continuously differentiable, strictly convex function over $\mathbb{R}^{N}$. Consider an $f$-proper partition. For any element $\mathcal{C}$ of this partition, the solution to $(P)$ completely determines $\mathcal{C}$.

Proof. Consider two elements $\mathcal{C}$ and $\mathcal{E}$ of the partition. We denote by $\left(k_{i}\right)_{i \in I}$ the characterization of $\mathcal{C}$ and by $\left(k_{i}^{\prime}\right)_{i \in I}$ the characterization of $\mathcal{E}$. We also write $Q=\sum_{i \in I} q_{i} \Psi_{i}$ for the unique solution to

$$
\left\{\begin{array}{l}
\text { minimize } f(v), \\
\text { over, } v \in \overline{\mathcal{C}}
\end{array}\right.
$$

and we assume that it is also the unique solution to

$$
\left\{\begin{array}{l}
\text { minimize } f(v), \\
\text { over, } v \in \overline{\mathcal{E}}
\end{array}\right.
$$

We need to prove that $\mathcal{C}=\mathcal{E}$.

Using the Kuhn-Tucker relation (2) for (4) and (5), we know there exist Kuhn-Tucker vectors $\left(\lambda_{i}\right)_{i \in I}$ and $\left(\lambda_{i}^{\prime}\right)_{i \in I}$, for (4) and (5) respectively, such that

$$
\begin{aligned}
\nabla f(Q) & =-\sum_{i \in I} \lambda_{i} \Psi_{i} \\
& =-\sum_{i \in I} \lambda_{i}^{\prime} \Psi_{i}
\end{aligned}
$$


This ensures that, since $\left(\Psi_{i}\right)_{i \in I}$ is a basis,

$$
\lambda_{i}=\lambda_{i}^{\prime}
$$

for all $i \in I$.

Now, since the partition is assumed to be $f$-proper, we obtain, again using Kuhn-Tucker relations,

$$
\left\{\begin{array}{cccc}
\text { if } \lambda_{i}=0, \quad \tau_{i, k_{i}}<q_{i}<\tau_{i, k_{i}+1} & \text { and } & \tau_{i, k_{i}^{\prime}}<q_{i}<\tau_{i, k_{i}^{\prime}+1}, \\
\text { if } \lambda_{i}>0, & q_{i}=\tau_{i, k_{i}+1} & \text { and } & q_{i}=\tau_{i, k_{i}^{\prime}+1} \\
\text { if } \lambda_{i}<0, & q_{i}=\tau_{i, k_{i}} & \text { and } & q_{i}=\tau_{i, k_{i}^{\prime}} .
\end{array}\right.
$$

It is then easy to prove that $\mathcal{C}=\mathcal{E}$.

Notice that the generalization of this statement to more general partitions (for instance when the partition is defined using a dictionary instead of a basis) is a very interesting and promising question. Answering this question is however not the purpose of this paper. Our purpose is rather to illustrate how such a property can be used.

Returning to image compression, observe that, to perform the correspondence between $Q$ and $\left(k_{i}\right)_{i \in I}$, both $q_{i}$ and $\lambda_{i}$ need to be tested. Notice also that, for the kind of partition we are considering, $\lambda_{i}$ is simply the $\mathrm{i}^{\text {th }}$ coordinates of $\nabla f(Q)$ and can therefore be computed.

\section{Coding images through an optimization problem}

\subsection{The theoretical and numerical coding procedure}

According to the results of the previous section, we can build the following coding/decoding procedure. Given a continuously differentiable and a strictly convex function $f$, defined over $\mathbb{R}^{N}$, an $f$-proper partition defined by a basis $\mathcal{B}=\left(\Psi_{i}\right)_{i \in I}$ and a quantization table $\left(\tau_{i, k}\right)_{i \in I, k \in \mathbb{Z}}$, we can code an image $u \in \mathbb{R}^{N}$ using the following steps:

1. Determine the sequence $\left(k_{i}\right)_{i \in I}$ defining $\mathcal{C}$, the element of the partition containing $u$. (This is numerically trivial with the proposed partition, but might be more difficult for other partitions.)

2. Determine the element $Q(u)=\sum_{i=1}^{N} q_{i} \Psi_{i}$ which solves $(P)$, for this $\mathcal{C}$. In general, this can be achieved by an iterative optimization algorithm. However, for computational time and convergence issues, having an analytical solution to $(P)$ is necessary.

3. Determine and encode $\left(J,\left(q_{j}\right)_{j \in J}\right)$, with

$$
J=\left\{i \in I, q_{i}=\tau_{i, k_{i}} \text { or } q_{i}=\tau_{i, k_{i}+1}\right\} .
$$

The encoding part of the process is beyond the scope of this paper. However, if the basis $\mathcal{B}$ (or more generally the partition) allows tree coding, our experiments make us believe it could still be applied.

The decoding is then performed by

1. Determine $Q(u)=\sum_{i=1}^{N} q_{i} \Psi_{i}$ which solves $\left(P^{\prime}\right)$, for $\mathcal{C}^{\prime}$ defined by

$$
\mathcal{C}^{\prime}=\left\{v=\sum_{i \in I} V_{i} \Psi_{i}, \forall j \in J, V_{j}=q_{j}\right\} .
$$

As for step 2 of the coding process, this can be achieved iteratively but should be achieved analytically.

2. Determine $\left(k_{i}\right)_{i \in I}$ from $Q(u)$. The correspondence between $\left(k_{i}\right)_{i \in I}$ and $Q(u)$ is given in (6). Moreover, in (6), for the partition which we are considering, the $\left(\lambda_{i}\right)_{i \in I}$ are the coordinates of $\nabla f(Q(u))$, in the basis $\mathcal{B}$. 


\subsection{Expressing quantization in this framework}

As stated in the introduction, given a basis $\mathcal{B}=\left(\Psi_{i}\right)_{i \in I}$ of $\mathbb{R}^{N}$, a quantization table $\left(\tau_{i, k}\right)_{i \in I, k \in \mathbb{Z}}$ and an image $u=\sum_{i \in I} U_{i} \Psi_{i}$, standard coders only code $\left(J,\left(k_{j}\right)_{j \in J}\right)$, where, for all $i \in I, k_{i}$ is such that

$$
\tau_{i, k_{i}} \leq U_{i}<\tau_{i, k_{i}+1}
$$

and

$$
J=\left\{i \in I, k_{i} \neq 0\right\}
$$

(We assume again that $\tau_{i, 0}<0<\tau_{i, 1}$, for all $i \in I$.)

The following propositions show that there is a one to one correspondence between the code $\left(J,\left(k_{j}\right)_{j \in J}\right)$ and the solution to an optimization problem of the form described in the previous section. In this sense, the compression method proposed in this paper generalizes the usual one.

Proposition 2 Given a basis $\mathcal{B}=\left(\Psi_{i}\right)_{i \in I}$ of $\mathbb{R}^{N}$ and $\left(\tau_{i, k}\right)_{i \in I, k \in \mathbb{Z}}$ a quantization table. We assume, for all $i \in I, \tau_{i, 0}<0<\tau_{i, 1}, \lim _{k \rightarrow \infty} \tau_{i, k}=-\lim _{k \rightarrow-\infty} \tau_{i, k}=+\infty$ and $\tau_{i, k}<\tau_{i, k+1}$, for all $k \in \mathbb{Z}$. For any $u=\sum_{i \in I} U_{i} \Psi_{i} \in \mathbb{R}^{N}$, and any $i \in I$, there is a one to one correspondence between $k_{i}$ (as determined by (7)) and $q_{i}\left(U_{i}\right)$, where

$$
q_{i}(t)= \begin{cases}\tau_{i, K_{i}} & , \text { if } K_{i}>0, \\ 0 & , \text { if } K_{i}=0, \\ \tau_{i, K_{i}+1} & , \text { if } K_{i}<0,\end{cases}
$$

for the unique $K_{i}$ such that

$$
\tau_{i, K_{i}} \leq t<\tau_{i, K_{i}+1} .
$$

Proof. For any $i \in I$, equation (8) obviously provides a way to determine $q_{i}\left(U_{i}\right)$, when $k_{i}$ is known.

Let us assume $q_{i}\left(U_{i}\right)$ is known. Observe that, for all $i \in I$, the sequence $\left(\tau_{i, k}\right)_{k \in \mathbb{Z}}$ is strictly increasing.

So, using (8), for any $i$ such that $q_{i}\left(U_{i}\right) \neq 0$, we can determine the unique $K_{i}^{\prime} \in \mathbb{Z}$ such that

$$
q_{i}\left(U_{i}\right)=\tau_{i, K_{i}^{\prime}} .
$$

We then set

$$
K_{i}\left(q_{i}\left(U_{i}\right)\right)= \begin{cases}K_{i}^{\prime} & , \text { if } q_{i}\left(U_{i}\right)>0, \\ 0 & , \text { if } q_{i}\left(U_{i}\right)=0, \\ K_{i}^{\prime}-1 & , \text { if } q_{i}\left(U_{i}\right)<0,\end{cases}
$$

It is not difficult to see that, for all $i \in I, K_{i}\left(q_{i}\left(U_{i}\right)\right)=k_{i}$, where $k_{i}$ is uniquely defined by (7).

Let us write

$$
\mathcal{C}_{u}=\left\{v=\sum_{i \in I} V_{i} \Psi_{i} \in \mathbb{R}^{N}, \text { for all } i \in I, \tau_{i, k_{i}} \leq V_{i}<\tau_{i, k_{i}+1}\right\},
$$

for the set of all $v \in \mathbb{R}^{N}$ having the same code as $u$. Then

$$
\overline{\mathcal{C}}_{u}=\left\{v=\sum_{i \in I} V_{i} \Psi_{i} \in \mathbb{R}^{N}, \text { for all } i \in I, \tau_{i, k_{i}} \leq V_{i} \leq \tau_{i, k_{i}+1}\right\},
$$

where $\overline{\mathcal{C}}_{u}$ denotes the closure of $\mathcal{C}_{u}$.

Proposition 3 Let $N>0$ be an integer, $\mathcal{B}=\left(\Psi_{i}\right)_{i \in I}$ be a basis of $\mathbb{R}^{N}$ and a quantization table

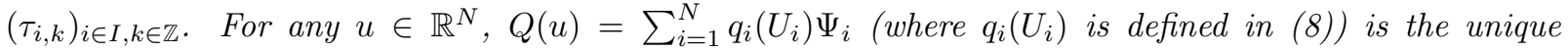
solution to

$$
\left(P_{1}\right):\left\{\begin{array}{l}
\text { minimize } f(v)=\sum_{i \in I} g_{i}\left(\left|V_{i}\right|\right) \\
\text { under the constraints } v=\sum_{i \in I} V_{i} \Psi_{i} \in \overline{\mathcal{C}}_{u} \text { (see (9)), }
\end{array}\right.
$$

where, for all $i, g_{i}$ is a non-decreasing function. 
Proof. It is easy to see that $\left(P_{1}\right)$ is equivalent to independently minimizing each $g_{i}\left(\left|V_{i}\right|\right)$ under the single constraint $\tau_{i, k_{i}} \leq V_{i} \leq \tau_{i, k_{i}+1}$. The solution to these 1D problems are obviously provided by $q_{i}\left(U_{i}\right)$.

Notice that if we follow the coding procedure described in the previous section, active constraints of $\left(P_{1}\right)$ exactly correspond to indexes in

$$
J=\left\{i \in I, U_{i}<\tau_{i, 0} \text { or } \tau_{i, 1} \leq U_{i}\right\} .
$$

So, according to Theorem 1 and Proposition $3, Q(u)$ is in fact solution to both $\left(P_{1}\right)$ and

$$
\left(P_{1}^{\prime}\right):\left\{\begin{array}{l}
\text { minimize } f(v)=\sum_{i \in I}\left|V_{i}\right|^{2} \\
\text { under the constraints } v \in \mathcal{C}_{u}^{\prime},
\end{array}\right.
$$

where

$$
\mathcal{C}_{u}^{\prime}=\left\{v=\sum_{i \in I} V_{i} \Psi_{i}, \forall j \in J, V_{j}=q_{j}\left(U_{j}\right)\right\} .
$$

The information we propose to code is therefore $\left(J,\left(q_{j}\left(U_{j}\right)\right)_{j \in J}\right)$. According to Proposition 2 this is precisely the information coded by the usual code $\left(J,\left(k_{j}\right)_{j \in J}\right)$.

\section{Conclusions}

In this paper, we proposed a method to compress images (or more generally data living in $\mathbb{R}^{N}$ ). It generalizes the current image compression standards. This generalization is made at the expense of numerical computations. We have not investigated which are the generalizations which are numerically tractable. At least, some functions $f$ and partitions built with bases provide numerically feasible compression models. We can only hope that future advances in numerical analysis allow more complex models to be tested.

If we leave numerical issues aside, we can expect improvements similar to those which have been obtained in image denoising, when comparing wavelet (or any other basis) thresholding and the solution to (1) (see [14, 2, 13, 12]). Indeed, in [14], a proposition similar to Proposition 3 makes a link between soft-thresholding and optimization models. The improvements obtained with (1) come from the choice of a better function $f$ and the introduction of redundancy in the data fidelity term. These are exactly the ingredients which are introduced, for image compression, in this paper :

- To transpose to the compression model under study the role of the redundant dictionary $\mathcal{D}$ in (1), we can consider a partition of $\mathbb{R}^{N}$ whose elements have more complex structures than hypercubes. In this case, the polyhedron defining the constraint in $(P)$ might have more faces of large codimension. These latter being opportunities to obtain small codes.

- The function $f$ determines the code of the elements of the partition. Heuristically, more codes (including short ones) are available in the regions where the level sets of $f$ are curved.

These are the reasons that make us believe such compression models might be useful.

Finally, beside the application to compression, this paper gives a new insight on the links between bases design and optimization theory. It underlines the role that the number of active constraints can play in the analysis of optimization models consisting in a projection onto a polyhedron (more precisely, a polytope). In this regard, the current paper is part a bigger project which includes $[15,16]$. We would like to underline that, in [16], another link is established between the number of active constraints (or more precisely, the dimension of the sub-space spanned by the active constraints) and sparse representation in a dictionary.

\section{References}

[1] F. Alter, S. Durand, and J. Froment. Adapted total variation for artifact free decompression of jpeg images. Journal of Mathematical Imaging and Vision, 23(2):199-211, Sept. 2005. 
[2] E. Candes and F. Guo. New multiscale transforms, minimum total variation synthesis:application to edge-preserving image reconstruction. Signal Processing, 82(11):1519-1543, 2002.

[3] T.F. Chan and H.M. Zhou. Optimal construction of wavelet coefficients using total variation regularization in image compression. Technical Report CAM 00-27, University of california Los Angeles, July 2000.

[4] S. S. Chen, D. L. Donoho, and M. A. Saunders. Atomic decomposition by basis pursuit. SIAM Journal on Scientific Computing, 20(1):33-61, 1999.

[5] A. Cohen, W. Dahmen, I. Daubechies, and R. De Vore. Harmonic analysis of the space bv. Revista Matematica Iberoamericana, 19:235-262, 2003.

[6] R. R. Coifman and A. Sowa. Combining the calculus of variations and wavelets for image enhancement. Applied and computational harmonic analysis, 9(1):1-18, 2000.

[7] R.R. Coifman, Y. Meyer, and M.V. Wickerhauser. Wavelet analysis and signal processing. In Wavelets and their Applications, pages 153-178. Jones and Barlett. B. Ruskai et al. eds, 1992.

[8] R.R. Coifman and M.V. Wickerhauser. Entropy-based algorithms for best basis selection. IEEE, Transactions on Information Theory, 38(2):713-718, March 1992.

[9] J. Darbon and M. Sigelle. A fast and exact algorithm for total variation minimization. Technical Report 2005D002, ENST, January 2005.

[10] J. Froment. Wavelets in Signal and Image Analysis, From Theory to Practice, chapter Image Compression Through Level Lines and Wavelet Packets. Kluwer Acad. Publ., Oct. 2001. A. Petrosian and F. Meyer Ed.

[11] N. Kingsbury. Image processing with complex wavelets. Phil. Trans. Roy. Soc. London A, 357:25432560, Sept. 1999. Special issue after the meeting "Wavelets : the key to intermittent information?".

[12] S. Lintner and F. Malgouyres. Solving a variational image restoration model which involves $l^{\infty}$ contraints. Inverse Problem, 20(3):815-831, June 2004.

[13] F. Malgouyres. Mathematical analysis of a model which combines total variation and wavelet for image restoration. Journal of information processes, 2(1):1-10, 2002. Available at http://www.math.univparis13.fr/ malgouy.

[14] F. Malgouyres. Minimizing the total variation under a general convex constraint for image restoration. IEEE, trans. on Image Processing, 11(12):1450-1456, Dec. 2002.

[15] F. Malgouyres. Projecting onto a polytope simplifies data distributions. Technical Report 2006-1, University Paris 13, January 2006. Available at : http://www.math.univ-paris13.fr/ malgouy.

[16] F. Malgouyres. Rank related properties for basis pursuit and total variation regularization. Technical Report ccsd-00020801, CCSD, March 2006. Available at : http://www.math.univparis13.fr/ malgouy.

[17] S. Mallat. A Wavelet Tour of Signal Processing. Academic Press, Boston, 1998.

[18] S. Mallat and Z. Zhang. Matching pursuits with time-frequency dictionaries. IEEE, Transactions on Signal Processing, 41(12):3397-3415, December 1993.

[19] F. Meyer and R. Coifman. Brushlets : A tool for directional image analysis and image compression. Applied and Computational Harmonic Analysis, 4:147-187, 1997.

[20] Y. Meyer. Ondelettes et opérateurs. Hermann, 1990.

[21] E. Le Pennec and S. Mallat. Bandelet image approximation and compression. SIAM, Multiscale Modeling and Simulation, 4(3):992-1039, 2005. 
[22] R.T. Rockafellar. Convex analysis. Princeton University Press, 1970.

[23] J. Romberg, M. Wakin, and R. Baraniuk. Multiscale Geometric Image Processing. In SPIE Visual Communications and Image Processing, Lugano, Switzerland, July 2003.

[24] J.L. Starck, E.J Candès, and D.L. Donoho. The curvelet transform for image denoising. IEEE, Transactions on image processing, 11(6):670-684, 2002. 